\title{
User selection protocols in FD PSP EH cooperative network over rayleigh fading channel: outage and intercept probability
}

\author{
Phu Tran Tin ${ }^{1}$, Minh Tran², Van-Duc Phan ${ }^{3}$, Hoang-Nam Nguyen ${ }^{4}$, Thanh-Long Nguyen ${ }^{5}$ \\ ${ }^{1}$ Wireless Communications Research Group, Faculty of Electrical and Electronics Engineering, Ton Duc Thang \\ University, Ho Chi Minh City, Vietnam \\ ${ }^{2}$ Optoelectronics Research Group, Faculty of Electrical and Electronics Engineering, Ton Duc Thang University, Ho Chi \\ Minh City, Vietnam \\ ${ }^{3}$ Center of Excellence for Automation and Precision Mechanical Engineering, Nguyen Tat Thanh University, Ho Chi \\ Minh City, Vietnam \\ ${ }^{4}$ Modeling Evolutionary Algorithms Simulation and Artificial Intelligence, Faculty of Electrical \& Electronics \\ Engineering, Ton Duc Thang University, Ho Chi Minh City, Vietnam \\ ${ }^{5}$ Center for Information Technology, Ho Chi Minh City University of Food Industry, Ho Chi Minh City, Vietnam
}

\section{Article Info \\ Article history: \\ Received Dec 12, 2018 \\ Revised Mar 1, 2019 \\ Accepted May 28, 2019}

\section{Keywords:}

Cooperative network Energy harvesting $(\mathrm{EH})$ Full duplex (FD)

Power splitting protocol (PSP)

User selection

\begin{abstract}
In this paper, we investigate the system performance in term of outage probability (OP) and intercept probability (IP) user selection protocols in full-duplex (FD) power splitting protocol (PSP) energy harvesting (EH) cooperative network over the Rayleigh fading channel. In this network, security and privacy issues are significant due to the possible eavesdropping by surrounding users. In this case, the security performance and reliable performance are represented by outage probability (OP) and intercept probability (IP), respectively. The power-splitting energy harvesting protocol is applied in our analysis. We rigorously derive the closed-form expressions of both OP and IP of the system and study the effect of various parameters. Finally, the Monte Carlo simulation results are also performed to confirm the correctness of all theoretical analysis derived.
\end{abstract}

Copyright (C) 2019 Institute of Advanced Engineering and Science. All rights reserved.

\section{Corresponding Author:}

Van-Duc Phan

Center of Excellence for Automation and Precision Mechanical Engineering,

Nguyen Tat Thanh University, Ho Chi Minh City, Vietnam

Email:pvduc@ntt.edu.vn

\section{INTRODUCTION}

Radio frequency (RF) energy transfer and harvesting has recently emerged as a promising way to extend the lifetime of energy-constrained wireless networks, especially when conventional energy harvesting techniques from renewable energy sources are not applicable. This technique enables wireless terminals to scavenge energy from RF signals broadcast by ambient/dedicated wireless transmitters to support their operation and information transmission. This new communication format has been termed wireless-powered communication (WPC) in the literature, which advocates the dual function of RF signals for both information delivery and energy transfer. In WPC, wireless terminals can avoid being interrupted by their batteries' depletion, which can thus be deployed more flexibly and maintained at a lower cost. In this sense, WPC has greater potential to sustain the network operation than its conventional battery-powered counterpart in the long run. Thanks to these inherent merits, WPC has been regarded as an indispensable and irreplaceable building block in a wide range of applications, e.g., RFID, wireless sensor networks, machine-to-machine communications, low-power wide-area networks, and the Internet of Things, and so on [1-5]. There are so many papers focus on the WPC direction. Such as some papers presented the process energy harvesting through the RF signals in cooperative wireless networks by a MIMO relay system the difference between the energy transfer and the information rates to provide the optimal source and relay precoding [6-8]. In the 
literature of the others, the authors investigated multi-user and multi-hop systems for simultaneous information and power transfer with a dual-hop channel with an energy harvesting relay; the transmission strategy depends on the quality of the second link [9-12]. Physical layer security has recently attracted a lot of attention to wireless communication community, especially when the traditional cryptography methods seems to be vulnerable to advanced attacks. Wyner introduced the idea of physical layer security in 1975 [13], but this topic has only been reconsidered recently $[14,15]$. The authors in [14] proposed an algorithm to maximize the secrecy sum rate of a two-way relay system by optimizing the relay beamforming vector and the transceivers powers under total power constraint. A physical layer-security scheme for an underlay relaybased cognitive radio network (CRN) that use s orthogonal frequency-division multiplexing (OFDM) as the medium access technique is proposed in [15], in which the secrecy rate is maximized subject to the constraints on transmission power and interference level at the primary users.

In this paper, we investigate the system performance (in term of outage probability (OP) and intercept probability (IP)) user selection protocols in full-duplex (FD) power splitting protocol (PSP) energy harvesting (EH) cooperative network over the Rayleigh fading channel. In this network, security and privacy issues are significant due to the possible eavesdropping by surrounding users. In this case, the security performance and reliable performance are represented by outage probability (OP) and intercept probability (IP), respectively. The power-splitting energy harvesting protocol is applied in our analysis. We rigorously derive the closed-form expressions of both OP and IP of the system and study the effect of various parameters. Finally, Monte Carlo simulation results are also performed to confirm the correctness of all theoretical analysis derived. The main contributions of the paper are summarized as follows:

1) The user selection protocols in full-duplex (FD) power splitting protocol (PSP) energy harvesting (EH) cooperative network over Rayleigh fading channel is proposed and investigated.

2) The closed-form expressions of OP and IP are derived. Furthermore, the influence of the main parameters on the system performance is demonstrated entirely by the Monte Carlo simulation.

The structure of this paper is proposed as follows. Sections II presents the system model of the relaying network. Sections III derives the system performance of the model system. Section IV provides numerical results and some discussions. Finally, Section V concludes the paper.

\section{SYSTEM MODEL}

In this paper, the system model is the full-duplex (FD) power splitting protocol (PSP) energy harvesting $(\mathrm{EH})$ cooperative network over Rayleigh fading channel (Figure 1). In this model, the information is transferred from the source $(\mathrm{S})$ to the multi-destination $\left(\mathrm{D}_{\mathrm{i}}\right)$, through energy constrained intermediate relay (R). In this network, there is an eavesdropper (E) who tries to listen illegally to the source data. We also assume that all of the nodes are equipped with a single antenna, and the data relaying is performed via two orthogonal time slots. The energy harvesting and information processing of the system model with FD PSP are proposed in Figure 2. In this scheme, $\mathrm{T}$ is the block time in which the source fully transmits the information data to the multi-destination. In this interval time, $\mathrm{R}$ harvests $\rho \mathrm{P}_{\mathrm{s}}$ energy from the source and use $(1-\rho) P_{s}$ energy to transfer information from $S$ to the multi-destination nodes, which $\rho$ is the power splitting factor $\rho \in(0,1)$. All the fading channels from $S$ to $R$ and $R$ to $D$ are proposed as the Rayleigh fading channels.

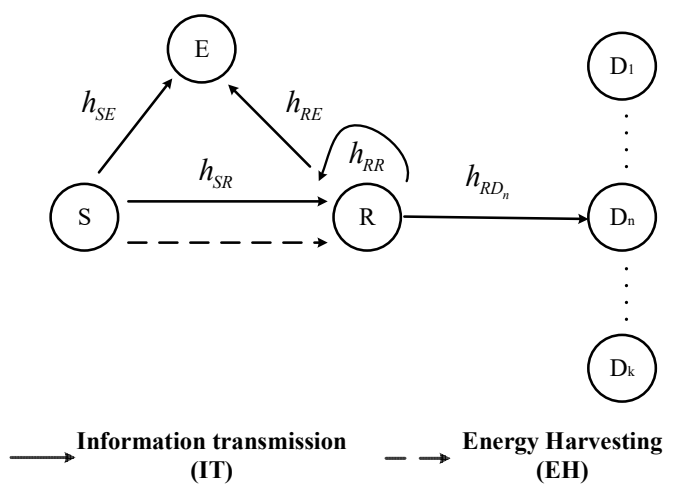

Figure 1. System model

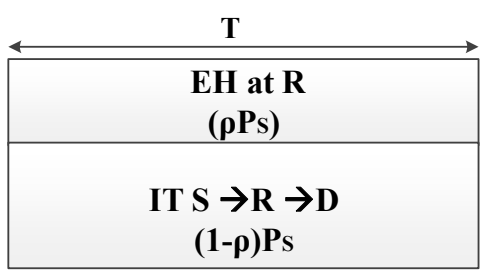

Figure 2. The power splitting protocol 


\section{THE SYSTEM PERFORMANCE}

During the first transmission phase, the received signal at the relay can be given by:

$$
y_{r}=\sqrt{(1-\rho)} h_{s r} x_{s}+h_{r r} x_{r}+n_{r}
$$

where $h_{s r}$ is the source to relay channel gain,

$h_{r r}$ is the loopback interference channel,

$x_{s}$ is the transmitted signal at the source,

$\mathrm{n}_{\mathrm{r}}$ is the additive white Gaussian noise (AWGN) with variance $\mathrm{N}_{0}$,

$0<\rho<1$ is PS ratio at the relay,

$x_{r}$ is the transmitted signal at the relay,

$\mathrm{E}\left\{\left|x_{s}\right|^{2}\right\}=P_{s}, \mathrm{E}\left\{\left|x_{r}\right|^{2}\right\}=P_{r}, \mathrm{E}\{\bullet\}:$ expectation operator,

$\mathrm{P}_{\mathrm{s}}$ is average transmit power at the source,

$\mathrm{P}_{\mathrm{r}}$ is average transmit power at the relay.

The harvested power at the relay can be obtained as

$$
P_{r}=\frac{E_{h}}{T}=\frac{\eta \rho P_{s}\left|h_{s r}\right|^{2} T}{T}=\eta \rho P_{s}\left|h_{s r}\right|^{2}
$$

where $0<\eta \leq 1$ is energy conversion efficiency.

The received signal at the $\mathrm{n}^{\text {rd }}$ destination can be expressed as the following

$$
y_{d_{i}}=h_{r d_{i}} x_{r}+n_{d_{i}}
$$

where, $h_{r d i}$ is the relay to the $\mathrm{i}^{\mathrm{rd}}$ destination channel gain, $\mathrm{n}_{\mathrm{di}}$ is the additive white Gaussian noise (AWGN) with variance $\mathrm{N}_{0}$.

In this model, we consider the decode-and-forward protocol. From (1), the signal to noise ratio (SNR) at the relay can be given by

$$
\gamma_{1}=\frac{(1-\rho)\left|h_{s r}\right|^{2} P_{s}}{\left|h_{r r}\right|^{2} P_{r}+N_{0}}
$$

Substituting (2) into (4) and using the fact that $N_{0}<<P_{s}$, in (4) can be rewritten as

$$
\gamma_{1} \approx \frac{1-\rho}{\eta \rho\left|h_{r r}\right|^{2}}
$$

From (2), the SNR at the destination can be calculated as

$$
\gamma_{2}=\frac{P_{r}\left|h_{r d_{i}}\right|^{2}}{N_{0}}=\frac{\eta \rho P_{s}\left|h_{s r}\right|^{2}\left|h_{r d_{i}}\right|^{2}}{N_{0}}=\eta \rho \gamma_{0}\left|h_{s r}\right|^{2}\left|h_{r d_{i}}\right|^{2}
$$

where we denote $\gamma_{0}=\frac{P_{s}}{N_{0}}$.

The final DF SNR can be given as

$$
\gamma_{D F}=\min \left(\gamma_{1}, \gamma_{2}\right)
$$

\subsection{User selection method.} follows

We propose the optimal user selection protocol in which the best selection user is selected as

Int J Pow Elec \& Dri Syst Vol. 10, No. 4, Dec 2019 : 2130 - 2137 
$\left|h_{r d}\right|^{2}=\max _{i=1,2, \ldots, K}\left(\left|h_{r d_{i}}\right|^{2}\right)$

In [16], the cumulative density function (CDF) of $\left|h_{r d}\right|^{2}$ can be given by

$$
F_{\left|h_{r d}\right|^{2}}(y)=\sum_{p=0}^{K}(-1)^{p} C_{K}^{p} \times e^{-p y / \lambda_{r d}}
$$

where $C_{K}^{p}=\frac{K !}{p !(K-p) !}, \lambda_{r d}$ is the mean of the random variable (RV) $\left|h_{r d}\right|^{2}$.

Then, the corresponding probability density function (PDF) can be obtained by

$$
f_{\left|h_{r d}\right|^{2}}(y)=\frac{1}{\lambda_{r d}} \sum_{p=0}^{K-1}(-1)^{p} C_{K-1}^{p} K \times e^{-(p+1) y / \lambda_{r d}}
$$

\subsection{Outage probability (OP).}

$$
O P=\operatorname{Pr}\left(\gamma_{D F}<z\right)=\operatorname{Pr}\left[\min \left(\gamma_{1}, \gamma_{2}\right)<z\right]=\operatorname{Pr}\left[\min \left(\frac{1-\rho}{\eta \rho\left|h_{r r}\right|^{2}}, \eta \rho \gamma_{0}\left|h_{s r}\right|^{2} \max \left|h_{r d_{i}}\right|^{2}\right)<z\right]
$$

By denoted as $\left|h_{r r}\right|^{2}=\omega_{1},\left|h_{s r}\right|^{2}=\omega_{2},\left|h_{r d}\right|^{2}=\max _{i=1,2, \ldots, K}\left(\left|h_{r d_{i}}\right|^{2}\right)=\omega_{3}$. In (10) can be rewritten as

$$
O P=\operatorname{Pr}\left[\min \left(\frac{1-\rho}{\eta \rho \omega_{1}}, \eta \rho \gamma_{0} \omega_{2} \omega_{3}\right)<z\right]
$$

where $z=2^{2 R}-1$ is threshold and R: the source rate. In (12) can be rewritten as

$$
O P=1-\operatorname{Pr}\left[\frac{1-\rho}{\eta \rho \omega_{1}} \geq z\right] \operatorname{Pr}\left[\eta \rho \gamma_{0} \omega_{2} \omega_{3} \geq z\right]
$$

we denote

$$
P_{1}=\operatorname{Pr}\left[\frac{1-\rho}{\eta \rho \omega_{1}} \geq z\right]=\operatorname{Pr}\left[\omega_{1} \leq \frac{\eta \rho z}{1-\rho}\right]=1-e^{-\frac{\eta \rho z}{\lambda_{r r}(1-\rho)}}
$$

where $\lambda_{r r}$ is the mean of RV $\left|h_{r r}\right|^{2}$ and

$$
\begin{aligned}
P_{2} & =\operatorname{Pr}\left[\eta \rho \gamma_{0} \omega_{2} \omega_{3} \geq z\right]=1-\operatorname{Pr}\left[\eta \rho \gamma_{0} \omega_{2} \omega_{3}<z\right]=1-\operatorname{Pr}\left[\omega_{2}<\frac{z}{\eta \rho \gamma_{0} \omega_{3}}\right] \\
& =1-\int_{0}^{\infty} F_{\omega_{2}}\left(\frac{z}{\eta \rho \gamma_{0} \omega_{3}} \mid \omega_{3}\right) f_{\omega_{3}}\left(\omega_{3}\right) d \omega_{3}=1-\int_{0}^{\infty}\left(1-e^{-\frac{z}{\lambda_{s r} \eta \rho \gamma_{0} \omega_{3}}}\right) f_{\omega_{3}}\left(\omega_{3}\right) d \omega_{3}
\end{aligned}
$$

Substituting (9) into (15), we have

$$
\begin{aligned}
P_{2} & =\int_{0}^{\infty} e^{-\frac{z}{\lambda_{s r} \eta \rho \gamma_{0} \omega_{3}}} \frac{1}{\lambda_{r d}} \sum_{p=0}^{K-1}(-1)^{p} C_{K-1}^{p} K \times e^{-(p+1) \omega_{3} / \lambda_{r d}} d \omega_{3} \\
& =\frac{1}{\lambda_{r d}} \sum_{p=0}^{K-1}(-1)^{p} C_{K-1}^{p} K \int_{0}^{\infty} e^{-\frac{z}{\lambda_{s r} \eta \rho \gamma_{0} \omega_{3}}} e^{-(p+1) \omega_{3} / \lambda_{r d}} d \omega_{3}
\end{aligned}
$$

Using Table of Integral Eq [3.324,1] in [17], the (16) can reformulate as

User selection protocols in FD PSP EH cooperative network over rayleigh fading ... (Van-Duc Phan) 


$$
P_{2}=2 \sum_{p=0}^{K-1}(-1)^{p} C_{K-1}^{p} K \sqrt{\frac{z}{\lambda_{r r} \lambda_{r d} \eta \rho \gamma_{0}(p+1)}} \times K_{1}\left(2 \sqrt{\frac{z(p+1)}{\lambda_{r r} \lambda_{r d} \eta \rho \gamma_{0}}}\right)
$$

Substituting (14), (17) into (13), we have

$$
O P=1-2\left[1-e^{-\frac{\eta \rho z}{\lambda_{r r}(1-\rho)}}\right] \sum_{p=0}^{K-1}(-1)^{p} C_{K-1}^{p} K \sqrt{\frac{z}{\lambda_{r r} \lambda_{r d} \eta \rho \gamma_{0}(p+1)}} \times K_{1}\left(2 \sqrt{\frac{z(p+1)}{\lambda_{r r} \lambda_{r d} \eta \rho \gamma_{0}}}\right)
$$

where $K_{v}(\bullet)$ is the modified Bessel function of the second kind and $\mathrm{v}^{\text {th }}$ order.

\subsection{Intercept probability (IP).}

The nodes $\mathrm{S}$ and $\mathrm{R}$ can randomly generate codebooks to confuse $\mathrm{E}$ from combining the received data with MRC (see randomize-and-forward (RF) method [18,19]). Therefore, the SNR obtained at E for decoding the source data can be formulated by

$$
\gamma_{E}=\max \left(\gamma_{S E}, \gamma_{R E}\right)
$$

where $\gamma_{S E}=\frac{P_{s}\left|h_{s e}\right|^{2}}{N_{0}}=\gamma_{0}\left|h_{s e}\right|^{2}, \gamma_{R E}=\frac{P_{r}\left|h_{r e}\right|^{2}}{N_{0}}=\gamma_{0} \eta \rho\left|h_{s r}\right|^{2}\left|h_{r e}\right|^{2}$

From (19), IP can be calculated precisely as (see [20])

$$
\begin{aligned}
I P & =\operatorname{Pr}\left(\gamma_{E} \geq z\right)=1-\operatorname{Pr}\left[\max \left(\gamma_{0}\left|h_{s e}\right|^{2}, \gamma_{0} \eta \rho\left|h_{s r}\right|^{2}\left|h_{r e}\right|^{2}\right)<z\right] \\
& =1-\operatorname{Pr}\left(\gamma_{0}\left|h_{s e}\right|^{2}<z\right) \operatorname{Pr}\left(\gamma_{0} \eta \rho\left|h_{s r}\right|^{2}\left|h_{r e}\right|^{2}<z\right) \\
P_{3} & =\operatorname{Pr}\left(\gamma_{0}\left|h_{s e}\right|^{2}<z\right)=1-e^{-\frac{z}{\lambda_{s e} \gamma_{0}}}
\end{aligned}
$$

where $\lambda_{s e}$ is the mean of RV $\left|h_{s e}\right|^{2}$. Then we have

$$
P_{4}=\operatorname{Pr}\left(\gamma_{0} \eta \rho\left|h_{s r}\right|^{2}\left|h_{r e}\right|^{2}<z\right)=\operatorname{Pr}\left(\gamma_{0} \eta \rho X<z\right)=\operatorname{Pr}\left(X<\frac{z}{\gamma_{0} \eta \rho}\right)=F_{X}\left(\frac{z}{\gamma_{0} \eta \rho}\right)
$$

where we denote $X=\left|h_{s r}\right|^{2}\left|h_{r e}\right|^{2}$.

Utilizing the result in [21], the CDF of $X$ can be shown as the below equation:

$$
F_{X}(x)=1-2 \sqrt{\frac{x}{\lambda_{s r} \lambda_{r e}}} K_{1}\left(2 \sqrt{\frac{x}{\lambda_{s r} \lambda_{r e}}}\right)
$$

where $K_{v}(\bullet)$ is the modified Bessel function of the second kind and $\mathrm{v}^{\text {th }}$ order.

From (23) and (22) can be computed as

$$
P_{4}=1-2 \sqrt{\frac{z}{\lambda_{s r} \lambda_{r e} \gamma_{0} \eta \rho}} K_{1}\left(2 \sqrt{\frac{z}{\lambda_{s r} \lambda_{r e} \gamma_{0} \eta \rho}}\right)
$$

where $\lambda_{s r}, \lambda_{r e}$ is the mean of RV $\left|h_{s r}\right|^{2},\left|h_{r e}\right|^{2}$, respectively.

Substituting (21), (24) into (20), the IP can be reformulated as

$$
I P=1-\left[1-e^{-\frac{z}{\lambda_{s e} \gamma_{0}}}\right]\left\{1-2 \sqrt{\frac{z}{\lambda_{s r} \lambda_{r e} \gamma_{0} \eta \rho}} K_{1}\left(2 \sqrt{\frac{z}{\lambda_{s r} \lambda_{r e} \gamma_{0} \eta \rho}}\right)\right\}
$$




\section{NUMERICAL RESULTS AND DISCUSSION}

In this section, the Monte Carlo simulation is used for validating the analytical expression in the above section. We consider a network with one source, one relay, and multi-destination, where source-relay and relay-destination distances are both normalized to unit value [22-25]. In Figure 3 and 4, OP and IP versus power splitting factor $\rho$ of the considered protocols is presented. Here, we set the main parameters as $\mathrm{R}=0.5$ bps, $\mathrm{P}_{\mathrm{s}} / \mathrm{No}=10 \mathrm{~dB}, \mathrm{~K}=1,2,4$. We also see from Figure 3 that the OP of the proposed system has a huge decrease in the interval of $\rho$ from 0 to 0.5 and has a massive increase while $\rho$ varies from 0.5 to 1 . The minimum value of the OP can be found at $\rho=0.5$. On another way, IP has a slight increase when $\rho$ increases from 0 to 1 . In the results, the simulation and theoretical results are in good agreement.

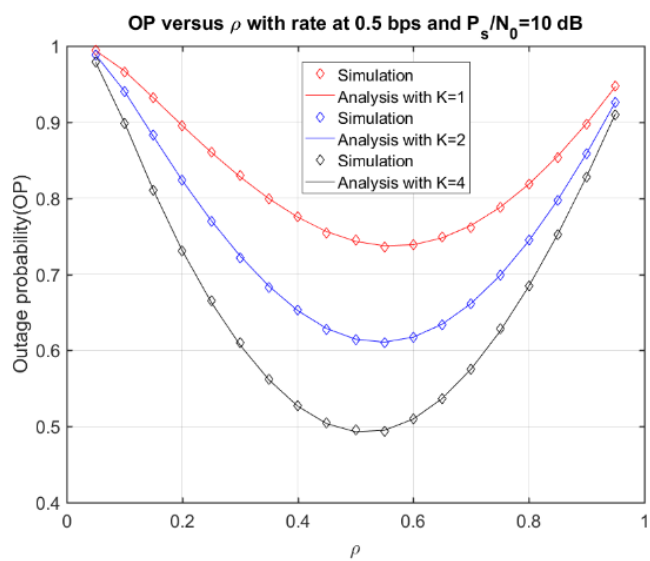

Figure 3. OP versus $\rho$.

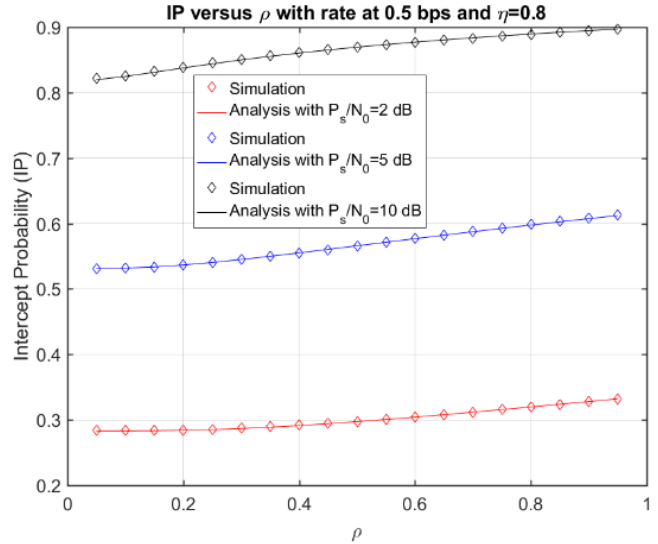

Figure 4. IP versus $\rho$.

Figure 5 and Figure 6 shows the outage probability and intercept probability of the proposed system versus the ratio $P_{s} / N_{0}$. The data rate is set to $R=0.5$ bps, $K=2, \rho=0.2,0.5,0.85$ for the OP case and $R=0.5$ bps, $\rho=0.5 \eta=0.3,0.6,0.85$, respectively. It can be observed that the simulation curve and the analytical curve for each case overlap together. That confirms the correctness of our analysis. It's also easily seen that when the ratio Ps/No increases, the outage probability decreases but the intercept probability increases. Moreover, the impact of $\mathrm{K}$ on the outage probability and $\eta$ on intercept probability is shown in Figure 7 and Figure 8 . Here, the ratio $\mathrm{Ps} / \mathrm{No}$ is set to $10 \mathrm{~dB}$. Again, the analytical solutions are in exact agreement with the simulation results. The outage probability tens to decrease as $\mathrm{K}$ increase. In contrast, the intercept probability increases with $\eta$ increases.

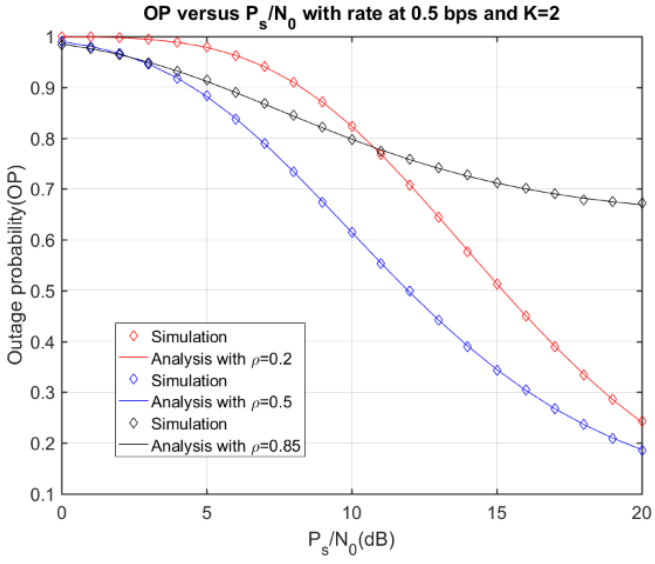

Figure 5. OP versus $\mathrm{P}_{\mathrm{s}} / \mathrm{N}_{0}$.

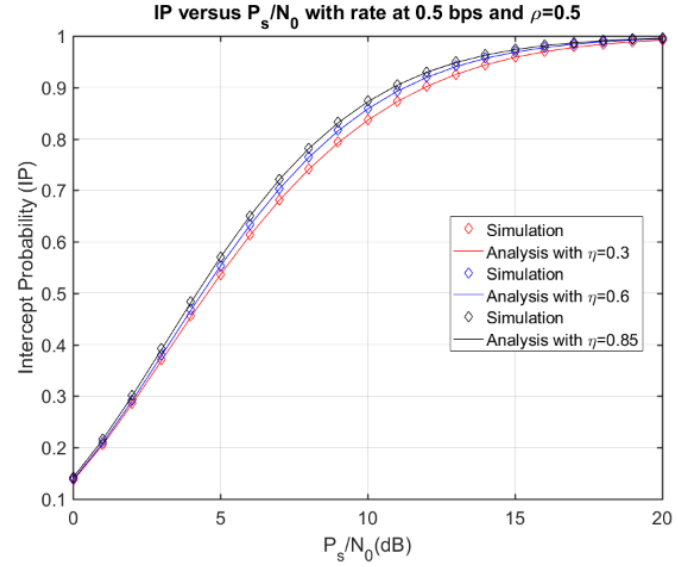

Figure 6. IP versus $\mathrm{P}_{\mathrm{s}} / \mathrm{N}_{0}$. 


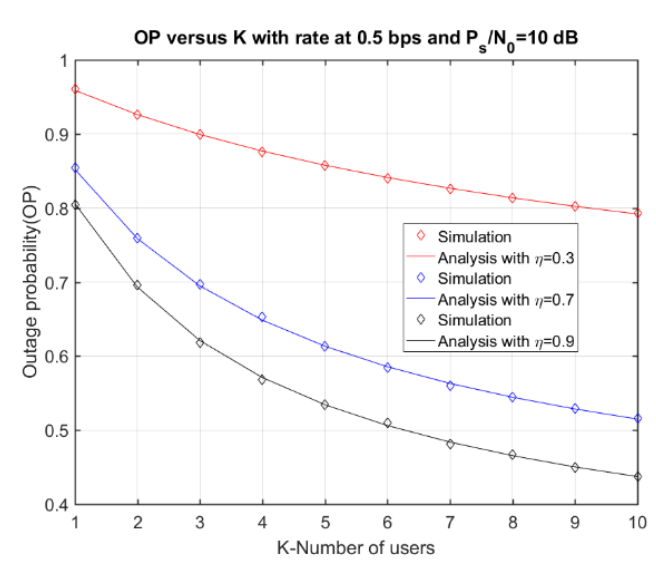

Figure 7. OP versus K

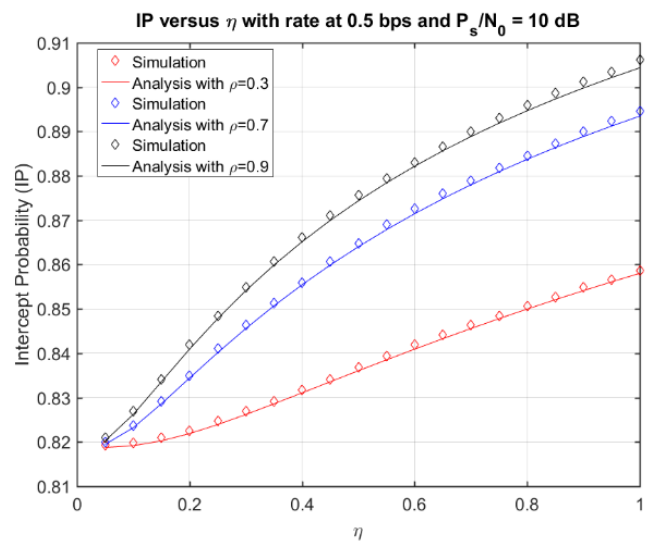

Figure 8. IP versus $\eta$.

\section{CONCLUSION}

In this paper, we investigate the system performance (in term of outage probability (OP) and intercept probability (IP)) user selection protocols in full-duplex (FD) power splitting protocol (PSP) energy harvesting $(\mathrm{EH})$ cooperative network over Rayleigh fading channel. In this network, the security performance and reliable performance are represented by outage probability (OP) and intercept probability (IP), respectively. We rigorously derive the closed-form expressions of both OP and IP of the system and study the effect of various parameters. Finally, Monte Carlo simulation results are also performed to confirm the correctness of all theoretical analysis derived.

\section{REFERENCES}

[1] Chen, He, Chao Zhai, Yonghui Li, and Branka Vucetic, "Cooperative Strategies for Wireless-Powered Communications: An Overview," IEEE Wireless Communications, vol. 25, no. 4, pp. 112-19, 2018. doi:10.1109/mwc.2017.1700245.

[2] Bi, S., Ho, C. K., \& Zhang, R., "Wireless powered communication: Opportunities and challenges," IEEE Communications Magazine, vol. 53, pp. 117-125, 2015.

[3] Niyato, D., Kim, D. I., Maso, M., \& Han, Z., "Wireless Powered Communication Networks: Research Directions and Technological Approaches," IEEE Wireless Communications, pp. 2-11, 2017.

[4] $\mathrm{Yu}, \mathrm{H}$. , Lee, H., \& Jeon, H., "What is 5G? Emerging 5G Mobile Services and Network Requirements. Sustainability," vol. 9, p. 1848, 2017.

[5] C. R. Valenta and G. D. Durgin, "Harvesting wireless power: Survey of energy-harvester conversion efficiency in far-field, wireless power transfer systems," IEEE Microw. Mag., vol. 15, no. 4, pp. 108-120, 2014.

[6] Sharma, V., \& Karmakar, P., "A Novel Method of Opportunistic Wireless Energy Harvesting in Cognitive Radio Networks," 2015 7th International Conference on Computational Intelligence, Communication Systems and Networks, 2015. doi:10.1109/cicsyn.2015.21

[7] A. M. Fouladgar and O. Simeone, "On the Transfer of Information and Energy in Multi-User Systems," IEEE Communications Letters, vol. 16, no. 11, pp. 1733-1736, 2012.

[8] Zhang, R., \& Ho, C. K., "MIMO Broadcasting for Simultaneous Wireless Information and Power Transfer. IEEE Transactions on Wireless Communications, vol. 12(5), 1989-2001, 2013. doi:10.1109/twc.2013.031813.120224

[9] Park, J., \& Clerckx, B., "Joint Wireless Information and Energy Transfer in a Two-User MIMO Interference Channel," IEEE Transactions on Wireless Communications, vol. 12(8), pp. 4210-4221, 2013.

[10] Chalise, B. K., Zhang, Y. D., \& Amin, M. G., "Energy harvesting in an OSTBC based amplify-and-forward MIMO relay system," 2012 IEEE International Conference on Acoustics, Speech and Signal Processing (ICASSP), 2012. doi:10.1109/icassp.2012.6288596

[11] Tutuncuoglu, K., \& Yener, A., "Cooperative energy harvesting communications with relaying and energy sharing," 2013 IEEE Information Theory Workshop (ITW), 2013. doi:10.1109/itw.2013.6691280

[12] Wyner, A.D., "The wire-tap channel," The Bell System Technical Journal, vol. 54(8), pp. 1355-1387, 1975.

[13] Wang, L., Wong K., Jin, S., Zheng, G., Jr., R.W.H., "A new look at physical layer security, caching, and wireless energy harvesting for heterogeneous ultra-dense networks," IEEE Communications Magazine, vol. 56, no. 6, pp. 49-55, 2018. https://doi.org/10.1109/MCOM.2018.1700439

[14] Obeed, M., Mesbah, W., "Efficient algorithms for physical layer security in two-way relay systems," Physical Communication, vol. 28, pp. 78 - 88, 2018.

[15] Shah, H.A., Koo, I., "A novel physical layer security scheme in ofdm-based cognitive radio networks," IEEE Access, vol. 6, pp. 29486-29498, 2018.

Int J Pow Elec \& Dri Syst Vol. 10, No. 4, Dec 2019 : 2130 - 2137 
[16] Tan N.Nguyen, Tran Trung Duy, Gia-Thien Luu, Phuong T.Tran and Miroslav Voznak,"Energy harvesting-based Spectrum Access With Incremental Cooperation, Relay Selection and Hardware Noises", RadioEngineering, Vol 26, No. 1, pp. 240-250, Apr. 2017.

[17] Table of Integrals, Series, and Products, 2015.

[18] J. Mo, M. Tao, Y. Liu, "Relay placement for physical layer security: A secure connection perspective," IEEE Commun. Lett, vol. 16 (6), pp. 878-881, 2012.

[19] T.T. Duy, P.N. Son, "Secrecy performances of multicast underlay cognitive protocols with partial relay selection and without eavesdropper's information," KSII Trans. Internet Inform. Syst., vol.9, no. 11, pp. 4623-4643, 2015.

[20] S. Jia, J. Zhang, H. Zhao, R. Zhang, "Relay selection for improved security in cognitive relay networks with jamming," IEEE Wireless Commun. Lett., vol. 6(5), pp. 662-665, 2017.

[21] Nguyen, Tan N., Phu Tran Tin, Phuong T. Tran, Tran Hoang Quang Minh, and Miroslav Voznak., "Power-Splitting Protocol in Power Beacon-assisted Energy Harvesting Full-Duplex Relaying Networks: Performance Analysis," 2018 11th IFIP Wireless and Mobile Networking Conference (WMNC), 2018. doi:10.23919/wmnc.2018.8480900.

[22] Tran Hoang Quang Minh, "Hybrid Time-Power Switching Protocol of Energy Harvesting Bidirectional Relaying Network: Throughput and Ergodic Capacity Analysis." TELKOMNIKA (Telecommunication, Computing, Electronics and Control), vol. 16, no. 5, p. 189. http://dx.doi.org/10.12928/telkomnika.v16i5.9118.

[23] Tin, Phu Tran, Tran Hoang Quang Minh, Tan N. Nguyen, and Miroslav Voznak, "System Performance Analysis of Half-Duplex Relay Network over Rician Fading Channel," TELKOMNIKA (Telecommunication, Computing, Electronics and Control), vol. 16, no. 1, p. 189, 2018. doi:10.12928/telkomnika.v16i1.7491.

[24] Rashid, Tarique, Sunil Kumar, Akshay Verma, Prateek Raj Gautam, and Arvind Kumar, "Pm-EEMRP: Postural Movement Based Energy Efficient Multi-hop Routing Protocol for Intra Wireless Body Sensor Network (IntraWBSN)," TELKOMNIKA (Telecommunication, Computing, Electronics and Control), vol. 16, no. 1, p. 166, 2018. doi:10.12928/telkomnika.v16i1.7318.

[25] Tin, Phu Tran, Minh Tran, Tan N. Nguyen, and Thanh-Long Nguyen, "System Performance Analysis of Hybrid Time-power Switching Protocol of EH Bidirectional Relaying Network in Amplify-and-forward Mode," Indonesian Journal of Electrical Engineering and Computer Science, vol. 14, no. 1, p. 118, 2019. doi:10.11591/ijeecs.v14.i1.pp118-126. 